\title{
Universal homogeneous causal sets
}

\author{
Manfred Droste \\ Institut für Informatik \\ Universität Leipzig \\ D-04109 Leipzig, Germany \\ email: droste@informatik.uni-leipzig.de
}

June 21, 2021

\begin{abstract}
Causal sets are particular partially ordered sets which have been proposed as a basic model for discrete space-time in quantum gravity. We show that the class $\mathfrak{C}$ of all countable past-finite causal sets contains a unique causal set $(U, \leq)$ which is universal (i.e., any member of $\mathfrak{C}$ can be embedded into $(U, \leq))$ and homogeneous (i.e., $(U, \leq)$ has maximal degree of symmetry). Moreover, $(U, \leq)$ can be constructed both probabilistically and explicitly.
\end{abstract}

\section{Introduction}

The causal set hypothesis asserts that the ultimate structure of space-time in quantum gravity is discrete, and that a fundamental relationship between points in space-time is causality, enabling us to say that $x$ is in the past of $y$. Such a structure can be naturally modelled by partially ordered sets $(S, \leq)$ which are locally finite (i.e., any interval $[x, y]$ between two points $x, y$ is finite) or which are even past-finite (i.e., the past of each point $x$ is finite). Locally finite partially ordered sets are called causal sets and have been investigated in detail, cf. e.g. [16. Moreover, an interesting sequential growth dynamics for finite causal sets was investigated in [15, 5, 4]: a finite causal set can be extended by a single element $z$ by adding it to the given partial order as a new maximal element (naturally, $z$ cannot be in the past of any given element). Continuing in this way, one obtains a countable past-finite causal set. Conversely, any countable past-finite causal set can be built up this way. For further work on this dynamics, see e.g. [1, 10, 12]

It is the goal of this paper to investigate the class of countable past-finite causal sets. We will show that there is a countable past-finite causal set $(U, \leq)$ which is universal and homogeneous. Here, universal means that $(U, \leq)$ contains an isomorphic copy of any countable past-finite causal set as a natural substructure. Homogeneous means that any isomorphism 
between two finite substructures (stems) of $(U, \leq)$ extends to an automorphism of $(U, \leq)$. Thus homogeneity intuitively says that $(U, \leq)$ has the highest possible degree of structural symmetry. Moreover, with these two properties, universality and homogeneity, $(U, \leq)$ is unique up to isomorphism in the class of all countable past-finite causal sets. Our proof employs the Fraïssé-Jónsson theorem well-known in model theory for constructions of homogeneous relational structures. We also give an explicit order-theoretic construction of the universal homogeneous past-finite causal set, and we show that the larger class of all countable causal sets (not requiring 'past-finite'), in contrast, does not contain a universal object.

In our second result, we will describe a probabilistic construction of the universal homogeneous past-finite causal set $(U, \leq)$. Probabilistic procedures for constructing one-point extensions of finite causal sets were crucial for [15, 10] and investigated in detail. Here we also propose a probabilistic construction. It is motivated by a classical construction of Erdös and Rényi [8] of the random graph (which is universal and homogeneous in the class of all countable graphs). More recently, a similar construction of the universal homogeneous countable partial order was given in [7]. If we employ the present probabilistic one-point extensions of finite causal sets successively, we obtain an infinite sequence of causal sets whose union is a countable past-finite causal set, the random past-finite causal set. Then we show that with probability 1, this random causal set is universal and homogeneous (hence unique up to isomorphism). Our construction of one-point extensions differs from the ones investigated in [15] in technical details, which we discuss briefly, and it would be interesting to investigate the relationship further. Finally, we describe a simple explicit number-theoretic construction of $(U, \leq)$. This is motivated by a classical construction of the random graph [13. We close with a short discussion.

\section{Universal causal sets}

First we establish some terminology. For a fuller introduction to causal sets, see [16].

A partially ordered set (poset) is a pair $(S, \leq)$ where $S$ is a set and $\leq$ is a binary relation on $S$ which is reflexive, antisymmetric and transitive. Let $(S, \leq)$ be a poset. For $x, y \in S$ we write $x<y$ to denote that $x \leq y$ and $x \neq y$. If $x<y$, we let $[x, y]=\{s \in S \mid x \leq s \leq y\}$, and for $x \in S$ let past $_{S}(x)=\{s \in S \mid s \leq x\}$. Then $(S, \leq)$ is called locally finite, if each interval $[x, y](x, y \in S, x<y)$ is finite, and past-finite, if $\operatorname{past}_{S}(x)$ is finite for each $x \in S$. Clearly, any past-finite poset is locally finite (but not conversely as seen by the set of negative integers with their natural order). A causal set (or causet) is a locally finite poset. If $X \subseteq S$ is a subset, let $\operatorname{past}_{S}(X)=\bigcup_{x \in X} \operatorname{past}(x)$. If $S$ is clear from the context, we also write past $(x)$ and past $(X)$. Then $X$ is called a stem of $S$, denoted $(X, \leq) \triangleleft(S, \leq)$ or $X \triangleleft S$, if $\operatorname{past}(X) \subseteq X$ (note that here, in slight generalization of [16], we do not require $X$ to be finite).

In [15, 16, constructions of countable causal sets $(S, \leq)$ are give as unions of chains of finite posets $\left(A_{1}, \leq_{1}\right) \triangleleft\left(A_{2}, \leq_{2}\right) \triangleleft \ldots$ Indeed, given such a chain, let $S=\bigcup_{i \in \mathbb{N}} A_{i}$, and for $x, y \in S$ put $x \leq y$ if for some $i \in \mathbb{N}$ we have $x, y \in A_{i}$ and $x \leq_{i} y$. Then $(S, \leq)$ is a 
past-finite countable poset and $A_{i} \triangleleft S$ for each $i \in \mathbb{N}$. Such constructions will also be crucial for this paper.

Let $(S, \leq)$ and $\left(S^{\prime}, \leq\right)$ be two posets. A mapping $f: S \rightarrow S^{\prime}$ is called an (order-) embedding of $(S, \leq)$ into $\left(S^{\prime}, \leq\right)$, if for any $x, y \in S$ we have $x \leq y$ iff $f(x) \leq f(y)$. Clearly, any embedding is one-to-one. An embedding $f:(S, \leq) \rightarrow\left(S^{\prime}, \leq\right)$ is called an isomorphism, if $f$ is onto, and a stem-embedding, if $f(S) \triangleleft S^{\prime}$. So, $f$ is a stem-embedding iff $f$ is an isomorphism from $(S, \leq)$ onto a stem of $\left(S^{\prime}, \leq\right)$. An isomorphism of $(S, \leq)$ onto itself is called an automorphism of $(S, \leq)$.

Let $\mathfrak{C}$ be a class (collection) of posets. A poset $(S, \leq) \in \mathfrak{C}$ will be called stem-universal in $\mathfrak{C}$, if each poset $(C, \leq) \in \mathfrak{C}$ is isomorphic to some stem in $(S, \leq)$, i.e. there exists a stem-embedding $f:(C, \leq) \rightarrow(S, \leq)$. We call $(S, \leq)$ homogeneous, if each isomorphism $f:(A, \leq) \rightarrow(B, \leq)$ between two finite stems of $(S, \leq)$ extends to an automorphism of $(S, \leq)$; equivalently, for any finite poset $(A, \leq)$ and any two stem-embeddings $f, f^{\prime}:(A, \leq) \rightarrow(S, \leq)$ there exists an automorphism $g$ of $(S, \leq)$ such that $f^{\prime}=g \circ f$ (We mention that in the literature there are other concepts called homogeneity. Our homogeneity, for instance, does not mean that $\operatorname{Aut}(S)$, the automorphism group of $(S, \leq)$, acts transitively on $S$.).

We say that $(S, \leq)$ realizes all one-point stem-extensions of finite stems of $S$, if whenever $(A, \leq),(B, \leq)$ are finite posets such that $(A, \leq) \triangleleft(S, \leq),(A, \leq) \triangleleft(B, \leq)$ and $|B|=|A|+1$, then there exists a stem-embedding $g:(B, \leq) \rightarrow(S, \leq)$ such that $\left.g\right|_{A}=\mathrm{id}_{A}$, the identity on $A$ (this condition includes the case $A=\emptyset$ ).

The first main goal of this paper will be the following result

Theorem 2.1. Let $\mathfrak{C}$ be the class of all past-finite countable causal sets.

(a) A poset $(U, \leq) \in \mathfrak{C}$ is stem-universal and homogeneous in $\mathfrak{C}$ iff $(U, \leq)$ realizes all onepoint stem-extensions of finite stems of $U$.

(b) There exists a stem-universal homogeneous past-finite causet $(U, \leq)$ in $\mathfrak{C}$. Moreover, $(U, \leq)$ is unique up to isomorphism with these properties.

One approach to the proof of Theorem 2.1 would be to use a category-theoretic generalization of the Fraïssé-Jónsson theorem from model theory, see [6], and to argue directly for past-finite causal sets and stem embeddings. In order to avoid the category-theoretic machinery needed for this, here we will base our argument on a more classical version of the Fraïssé-Jónsson theorem, cf. [9, 2, 11, which describes the existence of universal homogenous objects for various classes of e.g. relational structures. This also has the advantage of showing how to regard past-finite causal sets and stem-embeddings as relational structures and relational embeddings. For the convenience of the reader, we recall these notions. Let $\sigma=\left(n_{i}\right)_{i \in I}$ be a fixed $I$-indexed sequence of natural numbers $n_{i} \geq 1$ (a relational signature) denoting prescribed arities of relations. A tuple $\mathcal{A}=\left(A,\left(R_{i}\right)_{i \in I}\right)$ is called a $\sigma$-structure, if $A$ is a set (possibly empty) and $R_{i}$ is an $n_{i}$-ary relation on $A$, i.e. $R_{i} \subseteq A^{n_{i}}$, for each $i \in I$. Given two $\sigma$-structures $\mathcal{A}=\left(A,\left(R_{i}\right)_{i \in I}\right)$ and $\mathcal{B}=\left(B,\left(Q_{i}\right)_{i \in I}\right)$, a mapping $f: A \rightarrow B$ is called an embedding of $\mathcal{A}$ into $\mathcal{B}$, if $f$ is one-to-one and for each $i \in I$ and $x_{1}, \ldots, x_{n_{i}} \in A$ we have $\left(x_{1}, \ldots, x_{n_{i}}\right) \in R_{i}$ iff $\left(f\left(x_{1}\right), \ldots, f\left(x_{n_{i}}\right)\right) \in Q_{i}$. An embedding which is onto is called an 
isomorphism. An isomorphism of $\mathcal{A}$ onto itself is called an automorphism of $\mathcal{A}$. Further $\mathcal{A}$ is said to be a substructure of $\mathcal{B}$, if $A \subseteq B$ and $R_{i}=Q_{i} \cap A^{n_{i}}$ for each $i \in I$, i.e. $A \subseteq B$ and the identity mapping id : $A \rightarrow B$ is an embedding of $\mathcal{A}$ into $\mathcal{B}$; this is denoted by $\mathcal{A} \subseteq \mathcal{B}$.

Now let $\mathfrak{C}$ be a class (collection) of $\sigma$-structures. A structure $\mathcal{U} \in \mathfrak{C}$ is called universal in $\mathfrak{C}$, if for each $\mathcal{A} \in \mathfrak{C}$ there exists an embedding $f: \mathcal{A} \rightarrow \mathcal{U}$. Further, $\mathcal{U}$ is homogeneous, if each isomorphism $f: \mathcal{A} \rightarrow \mathcal{B}$ between two finite substructures $\mathcal{A}, \mathcal{B}$ of $\mathcal{U}$ with $\mathcal{A}, \mathcal{B} \in \mathfrak{C}$ extends to an automorphism of $\mathcal{U}$. The structure $\mathcal{U}$ realizes all finite extensions of finite substructures, if whenever $\mathcal{A}, \mathcal{B} \in \mathfrak{C}$ are finite structures such that $\mathcal{A} \subseteq \mathcal{U}$ and $\mathcal{A} \subseteq \mathcal{B}$, then there exists an embedding $g: \mathcal{B} \rightarrow \mathcal{U}$ such that $\left.g\right|_{A}=\mathrm{id}_{A}$. The class $\mathfrak{C}$ is said to be an $\omega$-class, if it satisfies the following conditions:

(0) Any $\mathcal{A} \in \mathfrak{C}$ is countable.

(1) Whenever $\mathcal{A} \in \mathfrak{C}$ and $\mathcal{B}$ is a structure isomorphic to $\mathcal{A}$, then $\mathcal{B} \in \mathfrak{C}$.

(2) If $\mathcal{A}_{1} \subseteq \mathcal{A}_{2} \subseteq \ldots$ are finite structures from $\mathfrak{C}$, forming a chain of substructures under inclusion, then their union $\mathcal{A}=\bigcup_{i \in \mathbb{N}} \mathcal{A}_{i}$ (whose domain and relations are defined as the union of the domain resp. corresponding relations of the structures $\mathcal{A}_{i}$ ) also belongs to $\mathfrak{C}$.

(3) If $\mathcal{A} \in \mathfrak{C}$ and $F$ is a finite subset of the domain of $\mathcal{A}$, then there exists a finite substructure $\mathcal{S} \subseteq \mathcal{A}$ with $\mathcal{S} \in \mathfrak{C}$ whose domain contains $F$.

An object $\mathcal{A} \in \mathfrak{C}$ is called weakly initial in $\mathfrak{C}$, if for each $\mathcal{B} \in \mathfrak{C}$ there exists an embedding $f: \mathcal{A} \rightarrow \mathcal{B}$. (Often, this is the empty or a singleton structure.) The class $\mathfrak{C}$ is said to have

- the joint embedding property, if for any $\mathcal{A}, \mathcal{B}$ there exists $\mathcal{C} \in \mathfrak{C}$ and embeddings $f$ : $\mathcal{A} \rightarrow \mathcal{C}, g: \mathcal{B} \rightarrow \mathcal{C}$

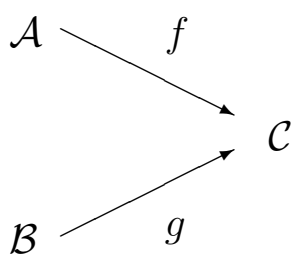

- the amalgamation property, if for any $\mathcal{A}, \mathcal{B}_{1}, \mathcal{B}_{2} \in \mathfrak{C}$ and embeddings $f_{i}: \mathcal{A} \rightarrow \mathcal{B}_{i}$ $(i=1,2)$ there exists $\mathcal{C} \in \mathfrak{C}$ and embeddings $g_{i}: \mathcal{B}_{i} \rightarrow \mathcal{C}(i=1,2)$ such that $g_{1} \circ f_{1}=g_{2} \circ f_{2}$, i.e. the subsequent diagram "commutes".

Theorem $2.2([\mathbf{2}, \mathbf{9}, 11])$. Let $\sigma$ be a relational signature and $\mathfrak{C}$ an $\omega$-class of $\sigma$-structures.

(a) Let $\mathfrak{C}$ contain a weakly initial structure. Then a structure $\mathcal{U} \in \mathfrak{C}$ is universal and homogeneous in $\mathfrak{C}$ if and only if $\mathcal{U}$ realizes all finite extensions of finite substructures. 


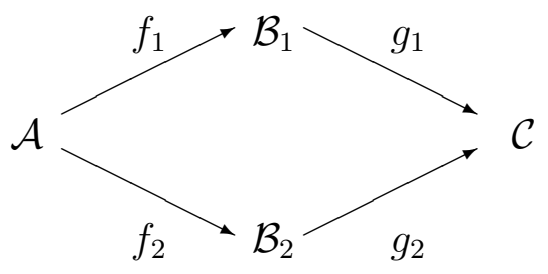

(b) The following are equivalent:

(1) $\mathfrak{C}$ contains a universal homogeneous structure $\mathcal{U}$.

(2) $\mathfrak{C}_{f i n}$, the class of all finite structures in $\mathfrak{C}$, has the joint embedding and the amalgamation property and contains up to isomorphism only countably many structures.

Moreover, in this case $\mathcal{U}$ is unique up to isomorphism.

We just note that the proof of Theorem [2.2(a) as well as of the uniqueness of $\mathcal{U}$ in part (b) employs a standard 'back-and-forth argument'. The construction of the universal homogeneous structure $\mathcal{U}$ given condition (2) of part (b) uses a suitable enumeration of all possible embeddings of the finite structures in $\mathfrak{C}$.

In order to be able to apply Theorem 2.2 to causal sets, we have to enrich them to relational structures such that stem-embeddings become relational embeddings as above. Given a poset $(A, \leq)$, we define its relational expansion to be $(A, \leq, \mathcal{R})$ where $\mathcal{R}=\left(R_{i}\right)_{i \geq 1}$ and $R_{i}=\{x \in A|| \operatorname{past}(x) \mid=i\}(i \geq 1)$. Hence $\leq$ is a binary and each $R_{i}$ a unary relation on $A$, so $(A, \leq, \mathcal{R})$ is a $\sigma$-structure for the signature $\sigma=(2,1,1,1, \ldots)$. Observe that if $A$ is finite, then each of the relations $R_{i}$ where $i>|A|$ is empty. Next we show that for past-finite causal sets, this expansion achieves our first goal:

Proposition 2.3. Let $(A, \leq)$ and $(B, \leq)$ be two past-finite causal sets and let $(A, \leq, \mathcal{R})$ resp. $(B, \leq, \mathcal{Q})$ be their relational expansions.

(a) $(A, \leq)$ is a stem of $(B, \leq)$ iff $(A, \leq, \mathcal{R})$ is a substructure of $(B, \leq, \mathcal{Q})$.

(b) Let $f: A \rightarrow B$ be a mapping. Then $f:(A, \leq) \rightarrow(B, \leq)$ is a stem-embedding iff $f:(A, \leq, \mathcal{R}) \rightarrow(B, \leq, \mathcal{Q})$ is an embedding of relational structures.

Proof. (a) We have $\mathcal{R}=\left(R_{i}\right)_{i \geq 1}$ and $\mathcal{Q}=\left(Q_{i}\right)_{i \geq 1}$ with $R_{i}=\left\{x \in A|| \operatorname{past}_{A}(x) \mid=i\right\}$ and $Q_{i}=\left\{y \in B|| \operatorname{past}_{B}(y) \mid=i\right\}(i \geq 1)$.

First, let $A \triangleleft B$. Let $x \in A$. By $A \triangleleft B$ we get $\operatorname{past}_{A}(x)=\operatorname{past}_{B}(x)$, so $x \in R_{i}$ iff $x \in Q_{i}$, for each $i \geq 1$. Hence $(A, \leq, \mathcal{R}) \subseteq(B, \leq, \mathcal{Q})$. Conversely, assume the latter. Let $x \in A$ and $y \in B$ with $y \leq x$. By assumption, we have $\operatorname{past}_{A}(x) \subseteq \operatorname{past}_{B}(x)$ and $\left|\operatorname{past}_{A}(x)\right|=\left|\operatorname{past}_{B}(x)\right| \in \mathbb{N}$, so past $A(x)=\operatorname{past}_{B}(x) \ni y$ showing $y \in A$. Hence $A \triangleleft B$.

(b) Straightforward by (a), using that the image of $A$ under $f$ is an isomorphic copy of $A$ and a stem resp. a substructure of $B$. 
This result allows us to translate all notions concerning stem-embeddings (like universality, homogeneity,...) into corresponding ones for the relational expansions and their embeddings.

Let $\mathfrak{C}$ be the class of all countable past-finite causal sets and let $\mathfrak{C}_{\text {rel }}$ be the collection of all relational expansions $(A, \leq, \mathcal{R})$ where $(A, \leq) \in \mathfrak{C}$. Next we give an easy application of Proposition 2.3 to show how this translation of notions works:

Remark 2.4. $\mathfrak{C}_{\text {rel }}$ is an $\omega$-class of $\sigma$-structures.

Proof. Conditions (0) and (1) of the definition of $\omega$-class are trivial. For (2), let

$\left(A_{1}, \leq, \mathcal{R}_{1}\right) \subseteq\left(A_{2}, \leq, \mathcal{R}_{2}\right) \subseteq \ldots$ be a chain of finite structures from $\mathfrak{C}_{\text {rel }}$, and let $\left(A, \leq, \mathcal{R}^{\prime}\right)$ be their union. Then $\left(A_{1}, \leq\right) \triangleleft\left(A_{2}, \leq\right) \triangleleft \ldots$ is a sequence of stems by Propositions 2.3 (a), and $(A, \leq)$ is a past-finite causal set whose expansion $(A, \leq, \mathcal{R})$ coincides with $\left(A, \leq, \mathcal{R}^{\prime}\right)$. Hence $\left(A, \leq, \mathcal{R}^{\prime}\right) \in \mathfrak{C}_{\text {rel }}$. To check $(3)$, let $(A, \leq, \mathcal{R}) \in \mathfrak{C}_{\text {rel }}$ and let $F$ be a finite subset of $A$. Then $\operatorname{past}(F)$ is a finite stem of $(A, \leq)$, so by Proposition 2.3 (a) its relational expansion is a finite substructure of $(A, \leq, \mathcal{R})$ belonging to $\mathfrak{C}_{\text {rel }}$.

Next we show:

Lemma 2.5. Let $(A, \leq),(B, \leq)$ be two finite posets such that $A \triangleleft B$. Then there is a sequence of stems

$$
A=A_{0} \triangleleft A_{1} \triangleleft \ldots \triangleleft A_{m}=B
$$

such that $\left|A_{i+1}\right|=\left|A_{i}\right|+1$ for each $i=0, \ldots, m-1$.

Proof. By induction on $|B \backslash A|$. Choose a minimal element $x$ of $B \backslash A$, and put $A_{1}=A \cup\{x\}$. Then $A \triangleleft A_{1} \triangleleft B$, and by induction we obtain a sequence of stems and one-point extensions from $A_{1}$ to $B$.

Now we can give the

Proof of Theorem 2.1. By Remark 2.4, $\mathfrak{C}_{\text {rel }}$ is an $\omega$-class of $\sigma$-structures.

(a) The empty structure is a weakly initial object of $\mathfrak{C}_{\text {rel }}$. Lemma 2.5 shows that if $\mathcal{U}$ realizes all one-point extensions of finite stems, it also realizes all finite stem-extensions of finite stems. Now the result is a translation of Theorem 2.2 (a).

(b) Again we use Proposition 2.3. We check condition (2) of Theorem 2.2 (b). It is clear that $\mathfrak{C}_{\text {rel }}$ contains up to isomorphism only countably many finite structures $(A, \leq, \mathcal{R})$, since if $A$ is finite, only finitely many of the relations $R_{i}$ are non-empty. It remains to show that the collection of finite posets satisfies the joint embedding and the amalgamation properties with respect to stem-embeddings. Since this collection contains the empty poset, it suffices to check the amalgamation property. For this, let $\left(A_{i}, \leq_{i}\right)(i=0,1,2)$ be three finite posets such that $\left(A_{0}, \leq_{0}\right) \triangleleft\left(A_{i}, \leq_{i}\right)$ for $i=1,2$. We may assume that $A_{0}=A_{1} \cap A_{2}$. Put $A=A_{1} \cup A_{2}$ and $\leq=\leq_{1} \cup \leq_{2}$, i.e. for $x, y \in A$ let $x \leq y$ iff either $x, y \in A_{1}$ and $x \leq_{1} y$ or $x, y \in A_{2}$ and $x \leq_{2} y$. Then $\leq$ is transitive, since if e.g. $x \leq_{1} y$ and $y \leq_{2} z$, we have $y \in A_{1} \cap A_{2}=A_{0}$ and $x \in A_{1}$. Since $A_{0} \triangleleft A_{1}$, we get $x \in A_{0}$ and $x \leq_{0} y$, so $x \leq_{2} y \leq_{2} z$ which implies $x \leq z$. 
Hence $\leq$ is a partial order on $A$. Observe that if $x \in A_{1} \backslash A_{0}$ and $y \in A_{2} \backslash A_{0}$, then neither $x \leq y$ nor $y \leq x$. Also note that if $x, y \in A_{1} \cap A_{2}$ and $x \leq_{1} y$, say, then this implies $x \leq_{0} y$, hence also $x \leq_{2} y$. We claim that $\left(A_{i}, \leq_{i}\right) \triangleleft(A, \leq)$ for $i=1,2$. By the remark just made, $\leq_{i}$ is just the restriction of $\leq$ to $A_{i}$. So, it only remains to show that $A_{i}$ is a stem of $(A, \leq)$.

Indeed, let $x \in A$ and $y \in A_{1}$ with $x \leq y$. We claim that $x \in A_{1}$. This is trivial if $x \leq_{1} y$. So let $x \leq_{2} y$. Then $x, y \in A_{2}$ and $y \in A_{0}$. Now $A_{0}$ is a stem of $\left(A_{2}, \leq\right)$, so $x \in A_{0} \subseteq A_{1}$. Hence $A_{1}$ is a stem of $(A, \leq)$ and for $A_{2}$ we argue analogously. This proves the amalgamation property.

Now the result follows from Theorem 2.2 (b).

Next we wish to describe the structure of the universal homogeneous past-finite causet $(U, \leq)$ further. A poset $S, \leq)$ is called directed, if for any $a, b \in S$ there is $c \in S$ with $a \leq c$ and $b \leq c$. Two elements $a, b \in S$ are incomparable, if neither $a \leq b$ nor $b \leq a$; this is denoted by $a \| b$. A subset $A \subseteq S$ is called an antichain, if any two elements of $A$ are incomparable. An element $x \in S$ is called maximal, if there is no $y \in S$ with $x<y$. Together with Theorem 2.1(a), the following provides an order-theoretic characterization of the structure of the universal homogeneous past-finite causal set.

Proposition 2.6. Let $(U, \leq)$ be a past-finite causal set. The following are equivalent;

(1) $(U, \leq)$ realizes all one-point stem-extensions of finite stems of $U$.

(2) (i) $(U, \leq)$ is directed, and

(ii) For any finite antichain $A \subseteq U$ (including the case that $A=\emptyset$ ) and any $y \in U \backslash A$ with $A \subseteq \operatorname{past}(y)$ there is $x \in U \backslash A$ such that $x \| y$ and $\operatorname{past}(x)=\operatorname{past}(A) \cup\{x\}$.

In this case, no element of $(U, \leq)$ is maximal.

Note that the condition past $(x)=\operatorname{past}(A) \cup\{x\}$ means that $a \leq x$ for each $a \in A$, and whenever $u \in U$ with $u<x$, then $u<a$ for some $a \in A$.

Proof. (1) $\rightarrow(2)$; To show (i), let $a, b \in U$. We claim that there is $c \in U$ with $a \leq c$ and $b \leq c$. Clearly, we may assume that $a \| b$. Let $A=\operatorname{past}(\{a, b\})$, choose an element $z \notin A$, and put $B=A \cup\{z\}$. We define a partial order $\leq^{*}$ on $B$ such that it extends the order $\leq$ on $A$ and $a \leq^{*} z, b \leq^{*} z$, so $z$ is the greatest element of $\left(B, \leq^{*}\right)$. Then $(A, \leq) \triangleleft\left(B, \leq^{*}\right)$, a one-point extension of the finite stem $A$ of $U$. Hence, there exists a stem-embedding $g:\left(B, \leq^{*}\right) \rightarrow(U, \leq)$ with $\left.g\right|_{A}=\mathrm{id}_{A}$. Now put $c=g(z)$ to obtain the chain.

For (ii), choose a finite antichain $A \subseteq U$ and $u \in U \backslash A$ with $A \subseteq \operatorname{past}_{U}(y)$. Put $A^{\prime}=\operatorname{past}_{U}(y)$, choose an element $z \notin A^{\prime}$, and put $B=A^{\prime} \cup\{z\}$. Define a partial order $\leq^{*}$ on $B$ such that it extends the order $\leq$ on $A$, such that each $x \in \operatorname{past}_{U}(A)$ satisfies $x \leq^{*} z$, but each $x \in A^{\prime} \backslash \operatorname{past}_{U}(A)$ is incomparable to $z$ in $\left(B, \leq^{*}\right)$. Then $\left(A^{\prime}, \leq\right) \triangleleft\left(B, \leq^{*}\right)$, a one-point extension of the finite stem $A^{\prime}$ of $U$. Again, there is a stem-embedding $g:\left(B, \leq^{*}\right) \rightarrow(U, \leq)$ with $\left.g\right|_{A^{\prime}}=\operatorname{id}_{A^{\prime}}$. Then $x=g(z)$ satisfies $x \notin A^{\prime}$ and $x \| z$, and by $g(B) \triangleleft U$ we obtain

$$
\begin{aligned}
\operatorname{past}_{U}(x) & =\operatorname{past}_{g(B)}(x)=g\left(\operatorname{past}_{B}(z)\right)=g\left(\operatorname{past}_{B}(A) \cup\{z\}\right) \\
& =\operatorname{past}_{g(B)}(A) \cup\{x\}=\operatorname{past}_{U}(A) \cup\{x\},
\end{aligned}
$$


as required.

$(2) \rightarrow(1)$; We first show that no element of $(U, \leq)$ is maximal. Let $y \in U$. Applying assumption (ii) to the empty antichain, we obtain $x \in U$ with $x \| y$. Now by (i) there is $z \in U$ with $x \leq z$ and $y \leq z$. Then $y<z$.

Now let $\left(A^{\prime} \leq\right)$ and $\left(B, \leq^{\prime}\right)$ be finite posets such that $\left(A^{\prime} \leq\right) \triangleleft(U, \leq),\left(A^{\prime} \leq\right) \triangleleft\left(B, \leq^{\prime}\right)$, and $B=A^{\prime} \cup\{z\}$, say. Let $A$ comprise all elements $a \in A^{\prime}$ maximal with respect to $a<^{\prime} z$ in $\left(B, \leq^{\prime}\right)$. So, $A$ is an antichain and $\operatorname{past}_{B}(z)=\operatorname{past}_{B}(A) \cup\{z\}$. Since $(U, \leq)$ is directed and $A^{\prime}$ is finite, there exists $y \in U$ with $a \leq y$ for each $a \in A^{\prime}$. Choose $y^{\prime} \in U$ with $y<y^{\prime}$; then $y^{\prime} \notin A^{\prime}$. Now by assumption (ii), there is $x \in U \backslash A$ such that in $(U, \leq)$ we have $x \| y^{\prime}$ and $\operatorname{past}_{U}(x)=\operatorname{past}_{U}(A) \cup\{x\}$. Define $g: B \rightarrow U$ by $\left.g\right|_{A^{\prime}}=\mathrm{id}_{A^{\prime}}$ and $g(z)=x$. By $x \| y^{\prime}$, we obtain $x \notin A^{\prime}$, and moreover, $x$ is incomparable to each element of $A^{\prime} \backslash \operatorname{past}_{U}(A)$. Hence $g$ is an embedding, and we show that $g(B) \triangleleft U$. We have $A^{\prime} \triangleleft U$, and so $\operatorname{past}_{U}(x)=\operatorname{past}_{U}(A) \cup\{x\} \subseteq A^{\prime} \cup\{x\}=g(B)$. Thus $g$ is a stem-embedding.

Next we wish to use Theorem 2.2(a) and Proposition 2.6 to give a direct construction (avoiding Theorem 2.2(b)) of the universal homogeneous past-finite causal set $(U, \leq$ ). We are thankful to an anonymous referee who pointed out this alternate construction.

Construction 2.7 (of the universal homogeneous past-finite causal set $(U, \leq)$ ). We construct a sequence of finite posets $\left(A_{1}, \leq_{1}\right) \triangleleft\left(A_{2}, \leq_{2}\right) \triangleleft \ldots$ as follows. Let $\left(A_{1}, \leq_{1}\right)$ be any singleton set, with the trivial order. Now let $i \in \mathbb{N}$ and assume we have constructed a finite poset $\left(A_{i}, \leq_{i}\right)$. Let $\mathcal{A}$ comprise all antichains in $\left(A_{i}, \leq_{i}\right)$, and choose pairwise different elements $x_{A}(A \in \mathcal{A})$ not belonging to $A_{i}$. Then put $A_{i+1}=A_{i} \cup\left\{x_{A} \mid A \in \mathcal{A}\right\}$, and define a partial order $\leq_{i+1}$ on $A_{i+1}$ such that it extends $\leq_{i}$ and for any $z \in A_{i+1}$, if $z \in$ past $_{A_{i}}(A) \cup\left\{x_{A}\right\}$ then $z \leq_{i+1} x_{A}$, but if $z \notin$ past $_{A_{i}}(A) \cup\left\{x_{A}\right\}$ then $z$ and $x_{A}$ are incomparable in $\left(A_{i+1}, \leq_{i+1}\right)$. Hence each element $x_{A}(A \in \mathcal{A})$ is maximal in $\left(A_{i+1}, \leq_{i+1}\right)$, and thus $\left(A_{i}, \leq_{i}\right) \triangleleft\left(A_{i+1}, \leq_{i+1}\right)$. Finally, let $(U, \leq)=\bigcup_{i \in \mathbb{N}}\left(A_{i+1}, \leq_{i+1}\right)$. Clearly, $\left(A_{i}, \leq_{i}\right) \triangleleft(U, \leq)$ for each $i \in \mathbb{N}$, and $(U, \leq)$ is a countable past-finite causal set.

Claim: $(U, \leq)$ is universal and homogeneous.

Proof. We show that $(U, \leq)$ satisfies condition (2) of Proposition 2.6 then this proposition and Theorem 2.1(a) imply the result. For condition (2)(i), let $a, b \in U$ with $a \| b$. Then $A=\{a, b\} \subseteq A_{i}$ for some $i \in \mathbb{N}$, and $x_{A} \in A_{i+1}$ satisfies $a \leq x_{A}$ and $b \leq x_{A}$. For condition (2)(ii), let $A \subset U$ be a finite antichain and $y \in U \backslash A$ with $A \subseteq$ past $_{U}(y)$. Again, $A \cup\{y\} \subseteq A_{i}$ for some $i \in \mathbb{N}$. Then $x_{A} \in A_{i+1}$ and by $A_{i+1} \triangleleft U$ and construction of $\leq_{i+1}$ we obtain

$$
\operatorname{past}_{U}\left(x_{A}\right)=\operatorname{past}_{A_{i+1}}\left(x_{A}\right)=\operatorname{past}_{A_{i+1}}(A) \cup\left\{x_{A}\right\}=\operatorname{past}_{U}(A) \cup\left\{x_{A}\right\} \text {. }
$$

Furthermore, since $y \notin$ past $_{A_{i+1}}(A)$, we get $x_{A} \| y$ in $\left(A_{i+1}, \leq_{i+1}\right)$ and in $(U, \leq)$, as required. Our claim follows.

This argument is more direct and intuitive than the previous one using Theorem 2.2 (b) with its proof. However, Theorem 2.2 puts the result into a general context, and checking the amalgamation property required for condition (2) of Theorem 2.2(b) was also uncomplicated. 
Next we mention two further structural properties of the universal homogeneous pastfinite causet $(U, \leq)$.

Corollary 2.8. Let $(U, \leq)$ be the universal homogeneous countable past-finite causet.

(a) For any finite subset $F \subseteq U,(U, \leq)$ is isomorphic to the poset $(U \backslash F, \leq)$. In particular, $(U, \leq) \cong(U \backslash \operatorname{past}(z), \leq)$ for each $z \in U$.

(b) For each $z \in U,(U, \leq)$ is isomorphic to $(\{u \in U \mid z<u\}$, $\leq)$, the proper future of $z$.

Proof. (a) We may assume that $F \neq \emptyset$. Trivially, $(U \backslash F, \leq)$ is a countable past-finite causet. We wish to check that $(U \backslash F, \leq)$ satisfies condition (2) of Proposition 2.6, then the result is immediate by Proposition [2.6 and Theorem 2.1. Since $(U, \leq)$ is directed and contains no maximal element, $(U \backslash F, \leq)$ is directed. So, let $A \subseteq U \backslash F$ be a finite antichain and $y \in(U \backslash F) \backslash A$ with $A \subseteq \operatorname{past}_{U \backslash F}(y)$. Again, since $(U, \leq)$ is directed and contains no maximal element, there is $y^{\prime} \in U \backslash F$ with $F \cup\{y\} \subseteq \operatorname{past}_{U}\left(y^{\prime}\right)$. By Proposition 2.6, for $(U, \leq)$, there is $x \in U \backslash A$ such that $x \| y^{\prime}$ and $\operatorname{past}_{U}(x)=\operatorname{past}_{U}(A) \cup\{z\}$. Then $x \notin F$ and neither $x \leq y$ nor $y \leq x$, and clearly past $U \backslash F(x)=$ past $_{U \backslash F}(A) \cup\{x\}$, as required for condition (ii). The final claim is immediate, since past $(z)$ is finite for each $z \in U$.

(b) Let $z \in U$ and $U^{\prime}=\{u \in U \mid z<u\}$. Again we show that $\left(U^{\prime}, \leq\right)$ satisfies condition (2) of Proposition 2.6. If the antichain $A$ chosen is empty, replace it by $\{z\}$. Then, and also in case $A$ is non-empty, apply condition (ii) for $(U, \leq)$ to obtain the element $x \in U^{\prime}$ as required.

Finally, we wish to show that the restriction to past-finite causets in Theorem 2.1 is essential:

Proposition 2.9. The class of all countable causets does not contain a universal causet.

Proof. We will exploit that this class also contains posets which are not past-finite. Suppose there was a countable causet $(U, \leq)$ such that each countable causet can be stem-embedded into $(U, \leq)$. Let $-\mathbb{N}$ denote the set of negative integers. We denote the natural partial order on $-\mathbb{N}$ by $\preceq$, so $n-1 \prec n$ for each $n \in-\mathbb{N}$. For each subset $A \subseteq-\mathbb{N}$ let $\left(S_{A}, \leq\right)$ be the causet obtained from $(-\mathbb{N}, \preceq)$ by replacing each element $n \in A$ by a 2 -element antichain $\left\{n, n^{*}\right\}$. That is, $S_{A}=-\mathbb{N} \cup\left\{n^{*} \mid n \in A\right\}, n$ and $n^{*}$ are incomparable for each $n \in A$, and $m<n^{*}$ iff $m<n$ iff $m \prec n(m \in-\mathbb{N}, n \in A)$, likewise for $m^{*}<n^{*}$ resp. $m^{*}<n$. By assumption, there is a stem-embedding $f_{A}:\left(S_{A}, \leq\right) \rightarrow(U, \leq)$. Let $x_{A}=f_{A}(-1)$. Then $f_{A}\left(S_{A}\right)$ is a stem of $U$, and $f_{A}\left(S_{A}\right)=\operatorname{past}\left(x_{A}\right)$.

As is easily seen, for $A, A^{\prime} \subseteq-\mathbb{N}$ we have $\left(S_{A}, \leq\right) \cong\left(S_{A^{\prime}}, \leq\right)$ iff $A=A^{\prime}$. Hence there are uncountably many non-isomorphic causets of the form $\left(S_{A}, \leq\right)$; these are isomorphic to the posets $\left(\operatorname{past}\left(x_{A}\right), \leq\right)$. But $U$ is countable and contains only countably many posets of the form $\operatorname{past}(x)(x \in U)$, a contradiction. 


\section{Probabilistic constructions}

In this section, we wish to provide probabilistic constructions of the universal homogeneous causal set $(U, \leq)$ of section 2 . Probabilistic constructions of causal sets were already investigated in [10, 15]. This employed successive one-point extensions ("generalized percolations") of finite posets; such extensions will also be used here. Suppose we are given a finite poset $(A, \leq)$ and want to extend it to a poset $(B, \leq)$ such that $B=A \cup\{z\}$, say, and $(A, \leq)$ is a stem in $(B, \leq)$. Then we just have to define the order relations between $z$ and the elements of $A$; moreover, since $A$ should become a stem of $B, z$ has to become a maximal element of $(B, \leq)$, thus for each $a \in A$ we can only put $a<z$ or $a \| z$, and this can be decided probabilistically. Let us now give the details of this construction for our case. We will discuss a technical difference to [10, 15] afterwards. For sake of concreteness, we will take the underlying set of our causet to be $\mathbb{N}$ (as in [15], but the construction would work for any countably enumerated set). Hence we will construct a partial order $\leq$ on $\mathbb{N}$. Recall that we write $x<y$ if $x \leq y$ and $x \neq y$. We denote the natural order on $\mathbb{N}$ by $\preceq$, i.e. $n \prec n+1$ for each $n \in \mathbb{N}$. Our basic construction will depend on a parameter $p \in[0,1]$.

Construction 3.1 (Probabilistic one-point extension of an enumerated finite poset). Let $p \in[0,1]$. Let $(A, \leq)$ be a finite poset with $A=\{1, \ldots, n\}$ for some $n \succeq 1$, and let $B=A \cup\{n+1\}$. Furthermore, letting $A_{j}=\{1, \ldots, j\}$ for $1 \preceq j \preceq n$, assume that $A_{1} \triangleleft \ldots \triangleleft A_{n}=A$. As noted before, we wish to extend the order $\leq$ to a partial order $\leq^{\prime}$ on $B$ such that $(A, \leq)$ is a stem of $\left(B, \leq^{\prime}\right)$. We proceed as follows. First choose, with equal probability, some $j \in\{1, \ldots, n\}$. If $j$ was chosen, this means that we will restrict ourselves to having $\operatorname{past}_{B}(n+1) \subseteq A_{j} \cup\{n+1\}$. We define a binary relation $R$ in $\{1, \ldots, n\} \times\{n+1\}$ as follows. Decide independently for each $i \in\{1, \ldots, j\}$ with probability $p$ that $(i, n+1) \in R$, and with probability $1-p$ that $(i, n+1) \notin R$. Now let $\leq^{\prime}$ on $B$ be the reflexive transitive closure of the relation $\leq \cup R$. That is, we have $n+1 \leq^{\prime} n+1$, for $x, y \in A$ we have $x \leq^{\prime} y$ iff $x \leq y$, and for any $x \in A$ we have $x \leq^{\prime} n+1$ iff there is $i \in\{1, \ldots, j\}$ such that $x \leq i$ and $(i, n+1) \in R$; in this case $x \in A_{j}$ by $A_{j} \triangleleft A$. Clearly, $\left(B, \leq^{\prime}\right)$ is a partial order, $n+1$ is maximal in $\left(B, \leq^{\prime}\right)$, $\operatorname{past}_{B}(n+1) \subseteq A_{j} \cup\{n+1\}$, and $(A, \leq) \triangleleft\left(B, \leq^{\prime}\right)$.

Next we wish to construct our random past-finite countable causal set structure on $\mathbb{N}$.

Construction 3.2 (of a probabilistic order $\leq$ on $\mathbb{N}$ ). Let $p \in[0,1]$. Let $A_{n}=$ $\{1,2, \ldots, n\}(n \in \mathbb{N})$. For $n=1$, put $1 \leq 1$. Now use Construction 3.1 to successively extend the order $\leq$ from $A_{n}$ to $A_{n+1}$. We obtain a sequence of stems $A_{1} \triangleleft A_{2} \triangleleft \ldots \triangleleft A_{n} \triangleleft \ldots$, and we put $(\mathbb{N}, \leq)=\bigcup_{n \in \mathbb{N}}\left(A_{n}, \leq\right)$. Then each $A_{n}$ is a stem of $(\mathbb{N}, \leq)$ and $(\mathbb{N}, \leq)$ is a past-finite causal set.

Next we will show:

Theorem 3.3. Let $p \in(0,1)$. With probability 1, the above construction produces a partial order $\leq$ on $\mathbb{N}$ such that $(\mathbb{N}, \leq)$ is a stem-universal homogeneous past-finite causal set. 
Proof. As noted before, $(\mathbb{N}, \leq)$ is a past-finite causal set. By Theorem 2.1 (a), it suffices to show that with probability $1,(\mathbb{N}, \leq)$ realizes all one-point stem-extensions of finite stems of $(\mathbb{N}, \leq)$. There are countably many such stem-extensions. Since the intersection of countably many events of probability 1 again has probability 1 , it suffices to consider an arbitrary fixed one-point stem-extension $A \triangleleft\left(B, \leq^{\prime}\right)$ with $B=A \cup\{y\}, A$ finite and $A \triangleleft(\mathbb{N}, \leq)$. We claim that with probability 1 there exists $z \in \mathbb{N}$ such that the mapping $g: B \rightarrow \mathbb{N}$ with $\left.g\right|_{A}=\operatorname{id}_{A}$ and $g(y)=z$ is a stem-embedding, i.e., for each $a \in A$ we have $a \leq^{\prime} y$ in $B$ iff $a \leq z$ in $\mathbb{N}$ and $A \cup\{z\} \triangleleft(\mathbb{N}, \leq)$.

For each $j \in \mathbb{N}$ let $A_{j}=\{1,2, \ldots, j\}$. Since $A$ is finite, there is $m \in \mathbb{N}$ such that $A \subseteq A_{m}$. Then $A \triangleleft A_{m}$. Now choose any integer $n \succeq m$ and consider the extension of the order $\leq$ from $A_{n}$ to $A_{n+1}$ given by Construction 3.1. We wish to compute a lower bound for the probability that we can put $z=n+1$ to obtain the required stem $A \cup\{z\} \triangleleft(\mathbb{N}, \leq)$. First, the probability that we choose the number $m$ from $\{1, \ldots, n\}$ is $\frac{1}{n}$. Then by Construction 3.1 for each $i \in A$, if $i<^{\prime} y$ in $\left(B, \leq^{\prime}\right)$ we put $(i, n+1) \in R$ with probability $p$, and if $i \|^{\prime} y$ we put $(i, n+1) \notin R$ with probability $1-p$; further with probability $1-p$ we put $(i, n+1) \notin R$ for each $i \in A_{m} \backslash A$. Hence, given $m$, there is a (small but) fixed $r>0$, depending only on the structure of $\left(A_{m}, \leq\right)$, on $A \triangleleft B$ and on $y$ (but not on $n$ ), such that at least with probability $r$ all $i \in A_{m}$ satisfy the above conditions, so $A \cup\{n+1\} \triangleleft A_{m} \cup\{n+1\} \triangleleft A_{n+1}$ and $n+1$ realizes the one-point stem extension of $A$ as required.

Thus the probability that in $\left(A_{n+1}, \leq\right)$, the structure of $\left(A_{m} \cup\{n+1\}, \leq\right)$ is as required is at least $r \cdot \frac{1}{n}$. Hence the probability that $n+1$ and $\left(A_{m} \cup\{n+1\}\right.$, $\left.\leq\right)$ do not satisfy these conditions is at most $1-\frac{r}{n}$. Consequently, the probability that no integer $n \succeq m, n+1$ and $\left(A_{m} \cup\{n+1\}\right)$ realizes this one-point extension of $A$ is $\prod_{n=m}^{\infty}\left(1-\frac{r}{n}\right)$. Since the series $\sum_{n=m}^{\infty} \frac{r}{n}$ diverges, we obtain $\prod_{n=m}^{\infty}\left(1-\frac{r}{n}\right)=0$. Hence with probability 1 , this one-point extension of $A$ is realized inside $(\mathbb{N}, \leq)$ as required, and the result follows.

We just note that the above 'intuitive' probabilistic statements can easily be made exact by a precise (but technical) definition of the employed probability space; this can be done analogously to the case of the random graph, cf. [8, 11, or to the procedure in [15, 5, 4] for constructing the probability space of completed labelled causets: The sample space of this probability space is the collection of all past-finite causets $(\mathbb{N}, \leq$ ) (hence the underlying set, $\mathbb{N}$, is fixed, but the order $\leq$ can vary), and Construction 3.1 yields a corresponding probability measure. Theorem 3.3 can then be rephrased by saying that in this probability space the collection of all causets which are universal and homogeneous has measure 1.

The above construction would work also for other probability distributions on $\{1, \ldots, n\}$, in Construction 3.1, than the uniform one. However, they would have to be chosen with some care in order to ensure the final argument in the proof of Theorem 3.3 .

Our construction of $(U, \leq)$ uses and depends on the given enumeration of $\mathbb{N}$, resp., in Construction [3.1, of the poset $A=\{1, \ldots, n\}$, and not only on the structure of the poset $(A, \leq)$ : After choosing $j \in\{1, \ldots, n\}$, we decided to possibly put $(i, n+1) \in R$ only for elements $i \in\{1, \ldots, j\}$. This is a bit unfortunate, since, in the notions of [15], it would correspond to some externally given time. Apart from this, our construction is very similar to the ones proposed in [10, 15]. It even shares with it a property of the "gregarious child 
transition", cf. [15, Lemma 2]:

Remark 3.4. Let $p \in[0,1]$, let $(A, \leq)$ be a finite poset with $A=\{1, \ldots, n\}$, and let $B=A \cup$ $\{n+1\}$. The probability to obtain by Construction 3.1 the poset $(B, \leq)$ with $(A, \leq) \triangleleft(B, \leq)$ and $n+1 \| a$ for each $a \in A$ depends only on the cardinality of $A$.

Proof. Given $j \in\{1, \ldots, n\}$, for each $i \in\{1, \ldots, j\}$ we have to put $(i, n+1) \notin R$. The probability of this equals $\frac{1}{n} \cdot \sum_{j=1}^{n}(1-p)^{j}$.

Furthermore, our construction apparently satisfies a weak form of the Bell causality condition, but not the condition of discrete general covariance of [10, 15]. It would be interesting to investigate this further. Also, this raises the question whether the universal homogeneous past-finite causet $(U, \leq)$ can be constructed without referring as in Construction 3.1 to a given enumeration, with positive probability, say. This would require a more intricate analysis of the probabilities of the finite posets occurring.

Finally, we wish to present also a number-theoretic representation of the universal homogeneous past-finite causet $(U, \leq)$. As underlying set, we take again the natural numbers $\mathbb{N}$.

Construction 3.5 (of a partial order $\leq$ on $\mathbb{N}$ ). We define a binary relation $R$ on $\mathbb{N}$ as follows. For any $j, n \in \mathbb{N}$, put $(j, n) \in R$ iff $j \prec n$ and in the unique ternary expansion of $n$ as a sum of distinct powers of $3,3^{j}$ occurs with coefficient 1 . That is, $(j, n) \in R$ iff

$$
n=3^{j}+\sum_{\substack{0 \leq i \preceq n \\ i \neq j}} x_{i} \cdot 3^{i}
$$

for some $x_{i} \in\{0,1,2\}$. Then let $\leq$ be the transitive reflexive closure of $R$.

Since $(j, n) \in R$ implies $j \prec n$, clearly $(\mathbb{N}, \leq)$ is a past-finite causal set, for $A_{n}=$ $\{1, \ldots, n\}$ we have $A_{n} \triangleleft \mathbb{N}$, and $n$ is a maximal element of $\left(A_{n}, \leq\right)$. We show:

Theorem 3.6. The poset $(\mathbb{N}, \leq)$ constructed above is a stem-universal homogeneous pastfinite causal set.

Proof. By Theorem 2.1 (a), it suffices to show that $(\mathbb{N}, \leq$ ) realizes all one-point extensions. So let $(A, \leq) \triangleleft(\mathbb{N}, \leq)$, with $A$ finite, $(A, \leq) \triangleleft\left(B, \leq^{\prime}\right)$ and $B=A \cup\{y\}$, say, with $y \notin A$. We claim that there exists $z \in \mathbb{N}$ such that the mapping $g: B \rightarrow \mathbb{N}$ with $\left.g\right|_{A}=\operatorname{id}_{A}$ and $g(y)=z$ is a stem-embedding. Choose $m \in \mathbb{N}$ such that $A \subseteq A_{m}$. Put

$$
z=\sum_{\substack{a \in A \\ a<\prime}} 3^{a}+2 \cdot 3^{m} .
$$

Then $z \notin A$ and $z$ is maximal in $(A \cup\{z\}, \leq)$. The construction of $\leq$ shows that if $a \in A$ with $a<^{\prime} y$, then $(a, z) \in R$, so $a<z$. Conversely, let $a \in \mathbb{N}$ and $a<z$. Since $\leq$ is the transitive reflexive closure of $R$, there is $a^{\prime} \in \mathbb{N}$ such that $a \leq a^{\prime}$ and $\left(a^{\prime}, z\right) \in R$. By definition of $R$ and of $z$, we immediately get $a^{\prime} \in A$ and $a^{\prime}<^{\prime} y$. Since $A \triangleleft \mathbb{N}$, we obtain $a \in A$ and $a<^{\prime} y$. It follows that $g$, defined as above, is an order-embedding of $\left(B, \leq^{\prime}\right)$ into $(\mathbb{N}, \leq)$ and that $g(B)$ is a stem of $\mathbb{N}$, as required. 


\section{Discussion}

We studied the class of all countable causal sets. In algebra, many classes of structures have been investigated with respect to the existence of universal or homogeneous objects. Most often, this completely depends on the given class of structures. Here, we could show that the class of all countable past-finite causets, in contrast to the class of all causets, contains a universal object. Somehow surprizingly, a random (intuitively: 'chaotic') construction produces, in the end, almost surely a universal causet bearing maximal degree of symmetry.

A basic idea behind causal set theory is that a manifold $M$ may emerge from a causal set $(S, \leq)$ by some sprinkling of a coarse-grained version of $(S, \leq)$ densely into $M$ (cf. [16]). Then it would be interesting to see how symmetry properties of $(S, \leq)$ are reflected in the structure of $M$. It is tempting to recall, in this context, Noether's close correspondence between symmetries and conservation laws. A bold question: Can conservation laws be founded on symmetry properties of causal sets and, ultimately, traced to some random constructions of causal sets?

\section{References}

[1] A. Ash and P. McDonald. Moment problems and the causal set approach to quantum gravity. J. Math. Phys., 44:1666-1678, 2003, [arXiv:gr-qc/0209020].

[2] J.L. Bell and A.B. Slomson. Models and Ultraproducts: An Introduction. North Holland, Amsterdam, 1969.

[3] L. Bombelli, J. Lee, D. Meyer, and R.D. Sorkin. Spacetime as a causal set. Phys. Rev. Lett., 59:521-524, 1987.

[4] G. Brightwell, F. Dowker, R.S. Garcia, J. Henson, and R.D. Sorkin. "Observables" in causal set cosmology. Phys. Rev., D67:084031, 2003, [arXiv:gr-qc/0210061].

[5] G. Brightwell, H.F. Dowker, R.S. García, J. Henson, and R.D. Sorkin. General covariance and the 'Problem of Time' in a discrete cosmology. pages 1-17. in K.G. Bowden, Ed., "Correlations", Proceedings of the ANPA 23 conference, 2001, Cambridge, England (Alternative Natural Philosophy Association, London, 2002), [arXiv:gr-qc/0202097].

[6] M. Droste and R. Göbel. Universal domains and the amalgamation property. Math. Struct. in Comp. Science, 3:137-159, 1993.

[7] M. Droste and D. Kuske. On random relational structures. Journal of Combinatorial Theory, A 102:241-254, 2003.

[8] P. Erdös and A. Rényi. Asymmetric graphs. Acta Math. Acad. Sci. Hungar., 14:295-315, 1963. 
[9] R. Fraissé. Sur l'extension aux relations de quelques propriétés des ordres. Ann. Sci. École Norm. Sup., 71:363-388, 1954.

[10] Nicholas Georgiou. A random binary order: a new model of random partial orders. CDAM research report, London School of Economics, U.K., 2003.

[11] W. Hodges. Model Theory. Cambridge University Press, Cambridge, 1993.

[12] X. Martin, D. O'Connor, D. Rideout, and R.D. Sorkin. On the "renormalisation" transformations induced by cycles of expansion and contraction in causal set cosmology. Phys. Rev., D63:084006, 2001, [arXiv:gr-qc/0009063].

[13] R. Rado. Universal graphs and universal functions. Acta Arith., 9:331-340, 1964.

[14] D.D. Reid. Discrete quantum gravity and causal sets. Canadian Journal of Physics, 79:1-16, 2001, [arXiv:gr-qc/9909075].

[15] D. Rideout and R. Sorkin. A classical sequential growth dynamics for causal sets. Phys. Rev., D61:024002, 2000, [arXiv:gr-qc/9904062].

[16] R.D. Sorkin. Causal Sets: Discrete Gravity. in the proceedings of the Valdivia Summer School, Valdivia, Chile, 2002, edited by A. Gomberoff and D. Marolf (to appear), [arXiv:gr-qc/0309009]. 